\title{
Infecção experimental de gerbis (Meriones unguiculatus) com nematódeos de ovinos: eficiência bionutricional
}

Helaíne Haddad Simões

$\mathrm{MACHADO}^{1}$

Francimar Fernandes

GOMES ${ }^{1}$

Francisco Carlos Rodrigues de OLIVEIRA ${ }^{1}$

Vagner Ricardo da Silva

FIUZA ${ }^{1}$

Edenio DETMANN²

\section{Correspondência para:}

HELAÍNE HADDADSIMÕES MACHADO

Laboratório de Sanidade Animal

Centro de Ciências e Tecnologias

Agropecuárias

UniversidadeEstadual do NorteFluminense Darcy Ribeiro

Av. Alberto Lamego, 2000 - Pq. Califórnia 28013-600 - Campos dos Goytacazes - RJ helaine@uenf.br

Recebido para publicação: 23/06/2005 Aprovado para publicação: 13/02/2006

1 - Laboratório de Sanidade Animal do Centro de Ciências e Tecnologias Agropecuárias da Universidade Estadual do Norte Fluminense Darcy Ribeiro, Campos dos Goytacazes - RJ

2 - Departamento de Zootecnia do Centro de Ciências Agrárias da Universidade Federal de Viçosa, Viçosa - MG

\section{Resumo}

O parasitismo por nematódeos gastrintestinais constitui uma importante causa de danos à saúde e de perdas econômicas na produção de pequenos ruminantes. O modelo laboratorial para estudos de nematódeos de ruminantes utilizando gerbis (Meriones unguiculatus) torna-se mais adequado quando estes animais são imunossuprimidos. Os corticosteróides são drogas freqüentemente usadas na imunossupressão de animais de biotério. $\mathrm{O}$ índice de eficiência bionutricional é considerado um parâmetro efetivo para avaliar os efeitos de tratamentos sobre a performance nutricional dos animais. O objetivo deste trabalho foi avaliar a eficiência bionutricional de gerbis imunossuprimidos e infectados experimentalmente com larvas de nematódeos de ovinos. Os resultados indicaram que os animais que receberam a droga apresentaram maior número de nematódeos à necropsia que o grupo de animais apenas infectados. Não foi observado efeito nocivo da infecção sobre a performance dos animais. Os animais não infectados e que receberam a droga metilprednisolona tiveram performance significativamente menor que os não infectados que não receberam a mesma.

\section{Introdução}

O parasitismo por nematódeos gastrintestinais constitui uma importante causa de danos à saúde e de perdas econômicas na produção de pequenos ruminantes ${ }^{1}$. Infecções experimentais em animais de laboratório utilizando nematódeos de ruminantes são realizadas para estudos da relação parasita-hospedeiro bem como testes in vivo de eficácia antihelmíntica ${ }^{2,3}$. Segundo Coutinho ${ }^{4}$, o modelo utilizando o coelho (Oryctolagus cuniculus) é considerado bom, entretanto, requer espaço e custo de manutenção mais onerosos.

Gerbis (Meriones unguiculatus) e ratos Wistar (Rattus novergicus) já foram utilizados com sucesso; porém, com a necessidade de imunossupressão prévia ${ }^{2,5,3,3}$. Outras pesquisas foram feitas utilizando gerbis como modelo para infecções concomitantes de Haemonchus contortus e Trichostrongylus colubriformis ${ }^{2}$ e para avaliar desenvolvimento e duração da imunidade adquirida contra estes parasitas ${ }^{6}$.

Os corticosteróides constituem drogas amplamente utilizadas na imunossupressão de animais de laboratório para fins de pesquisas em parasitologia ${ }^{7,8}$. Em estudo da infecção experimental de Strongyloides stercoralis em gerbis, utilizou-se a metilprednisolona como droga imunossupressora?. Além disso, pesquisas relativas ao tratamento com metilpredni-solona para pacientes em fase de recuperação de distúrbios neurológicos e isquemia cerebral, utilizando gerbis como modelo, foram realizadas ${ }^{10,11}$.

A eficiência bionutricional (EBN) é 
citada como um método efetivo para avaliar o efeito de tratamentos sobre o desempenho nutricional de animais, considerada mais sensível que a conversão alimentar ${ }^{12}$. Estudo anterior inferindo sobre a EBN de gerbis experimentalmente infectados com oocistos esporulados de Cystoisospora felis comprovou os efeitos deletérios deste coccídio, bem como a susceptibilidade da espécie $M$. unguiculatus ao mesmo ${ }^{13}$.

Este trabalho teve como objetivo infectar experimentalmente gerbis imunossuprimidos e não imunossuprimidos com nematódeos de ovinos para avaliar a EBN e, dessa maneira, contribuir para o conhecimento da relação parasita-hospedeiro, além de verificar o efeito da imunossupressão na EBN.

\section{Materiais e Métodos}

Foram utilizados 20 gerbis, obtidos de colônia mantida pelo Setor de Doenças Infecto-Contagiosas e Parasitárias do Laboratório de Sanidade Animal (LSA) da Universidade Estadual do Norte Fluminense Darcy Ribeiro (UENF). Os gerbis foram distribuídos em quatro grupos com cinco animais cada, um grupo com animais infectados e imunossuprimidos; um grupo com animais infectados e não-imunossuprimidos, além de dois grupos controle, um de animais não-infectados e imunossuprimidos e outro de não-infectados e nãoimunossuprimidos.

As larvas infectantes $\left(\mathrm{L}_{3}\right)$ que constituíram os inóculos foram obtidas por coprocultura a partir de fezes de ovinos oriundos da Região de Campos dos Goytacazes, RJ. As larvas foram imobilizadas com lugol, identificadas e contadas em alíquotas de $100 \mathrm{ml}$ da suspensão, em triplicata. Foram identificados os gêneros Haemonchus, Trichostrongylus, Cooperia, Strongyloides, Bunostomum. Os inóculos contendo os parasitas dos gêneros previamente identificados foram padronizados de modo que cada animal recebesse a dose média de $1,0 \times 10^{3} \mathrm{~L}_{3}$ suspensas em água destilada. A imunossupressão consistiu na aplicação por via intramuscular de acetato de metilprednisolona (Depo-Medrol ${ }^{\circledR}$, Pharmacia), na dose de $0,1 \mathrm{ml}$ por animal, correspondendo a $4,0 \mathrm{mg}$ do princípio ativo, com repetição após 21 dias.

Os animais foram mantidos em área coberta, recebendo ração própria para a espécie e água ad libitum. As variáveis ganho de peso e consumo de ração foram avaliadas semanalmente, durante oito semanas, desde a infecção. A infecção foi confirmada através de exames coproparasitológicos e recuperação de helmintos adultos à necropsia, 58 dias após a infecção (DAI). Os conteúdos de estômago e intestinos foram lavados sucessivas vezes e os helmintos recuperados foram fixados em formol tamponado a 10\%, para em seguida serem identificados e contados.

Os resultados foram analisados estatisticamente através do teste $\mathrm{t}(\mathrm{P}<0,05)$ para comparação de médias, utilizando-se delineamento inteiramente casualizado, em esquema fatorial $2 \times 2(a=0,10)$. Previamente, os dados relativos às variáveis consumo $\mathrm{e}$ ganho foram submetidos à avaliação quanto à homogeneidade de variâncias pelo teste de Bartlett e normalidade dos erros pelo teste de Lilliefors ${ }^{14}$. Devido à não-normalidade, os dados de ganho foram submetidos à transformação logarítmica. As estimativas de EBN foram obtidas segundo métodos descritos na literatura ${ }^{12}$.

\section{Resultados e Discussão}

Os resultados relativos ao efeito do parasitismo em relação a EBN e do percentual das espécies de nematódeos de ovinos recuperados em relação ao efeito da imunossupressão de gerbis podem ser observados nas tabelas 1 e 2 , respectiva-mente.

De acordo com os resultados da tabela 1 , pode-se observar que o ganho de peso e a EBN dos animais do grupo que recebeu o corticosteróide foram estatisticamente menores em relação ao grupo dos animais não medicados, durante o período experimental. 
Desta forma, pode-se inferir que a administração de metilprednisolona foi capaz de exercer efeito significativo sobre a EBN dos animais, retardando o desenvolvimento dos mesmos. Estes resultados estão de acordo com a literatura, no que se refere às contra-indicações do uso de altas dosagens ou de tempo prolongado de terapia com corticosteróides ${ }^{15}$. A análise dos resultados permitiu afirmar, também, que a infecção com larvas de nematódeos de ovinos não influenciou o desempenho dos animais, visto que a EBN dos grupos inoculados (imunossuprimidos e não imunossuprimi-dos) não foi significantemente diferente em relação aos grupos não inoculados, ao longo do período estudado. Em estudo do desenvolvimento da imunidade em gerbis infectados com $T$. colubriformis, Ziam et $\mathrm{al}^{6}$, verificaram altos níveis de $\operatorname{IgA}$ intestinal, bem como fora significativamente maior a resposta sistêmica de IgM e IgG quando comparada à de um grupo que não fora desafiado com a infecção. Desta forma, novos estudos devem ser realizados a fim de verificar se a não influência da infecção sobre a EBN pode ser atribuída a infectividade larvar ou ao mecanismo compensatório de resposta imunológica do hospedeiro.

Visto que o gerbil é um animal de laboratório bastante utilizado como modelo experimental e que muitas vezes requer imunossupressão prévia à infecções experimentais ${ }^{7}$, faz-se presente uma maior atenção aos possíveis efeitos negativos para o desenvolvimento desses animais provocados por corticóides.

Tabela 1 - Médias de quadrados mínimos, coeficientes de variação e níveis descritivos de probabilidade para o erro tipo I para as variáveis consumo e ganho médios diários e eficiência bionutricional (EBN), de acordo com os efeitos de infecção e imunossupressão. Campos dos Goytacazes, Junho de 2005

\begin{tabular}{|c|c|c|c|c|}
\hline \multirow{2}{*}{\multicolumn{2}{|c|}{ Médias de Tratamentos }} & \multicolumn{3}{|c|}{ Variável } \\
\hline & & Consumo $^{1}$ & Ganho $^{2}$ & $\mathrm{EBN}^{3}$ \\
\hline \multirow{2}{*}{ Infectados } & $\begin{array}{c}\text { Com } \\
\text { Cmunosupressão }\end{array}$ & 9,815 & $-1,8505$ & $-0,0001$ \\
\hline & $\begin{array}{c}\text { Sem } \\
\text { Imunossupressão }\end{array}$ & 7,590 & $-2,1319$ & $-0,2178$ \\
\hline Não- & $\begin{array}{c}\text { Com } \\
\text { Imunossupressão }\end{array}$ & 7,622 & $-2,5421$ & $-0,3434$ \\
\hline Infectados & $\begin{array}{c}\text { Sem } \\
\text { Imunossupressão }\end{array}$ & 8,907 & $-1,4208$ & 0,0803 \\
\hline \multicolumn{5}{|c|}{ Valor P - Efeitos Principais } \\
\hline \multicolumn{2}{|c|}{ Infecção } & 0,6895 & 0,9795 & 0,8800 \\
\hline \multicolumn{2}{|c|}{ Imunossupressão } & 0,6684 & 0,2805 & 0,4961 \\
\hline \multicolumn{2}{|c|}{ Infecção X Imunossupressão } & 0,1270 & 0,0835 & 0,0493 \\
\hline \multicolumn{2}{|c|}{ Coeficiente de Variação (\%) } & 24,8 & 37,3 & 256,8 \\
\hline \multicolumn{5}{|c|}{ Valor P - Contrastes } \\
\hline \multicolumn{2}{|c|}{$\begin{array}{l}\text { Efeito da Infecção com } \\
\text { Imunossupressão }\end{array}$} & - & 0,2392 & 0,1453 \\
\hline \multicolumn{2}{|c|}{$\begin{array}{l}\text { Efeito da Infecção sem } \\
\text { Imunossupressão }\end{array}$} & - & 0,1727 & 0,1496 \\
\hline \multicolumn{2}{|c|}{$\begin{array}{l}\text { Efeito da Imunossupressão com } \\
\text { Infecção }\end{array}$} & - & 0,5767 & 0,2827 \\
\hline \multicolumn{2}{|c|}{$\begin{array}{l}\text { Efeito da Imunossupressão sem } \\
\text { Infecção }\end{array}$} & - & 0,0677 & 0,0786 \\
\hline
\end{tabular}

1/g/animal/dia. 2/logaritmo natural de g/animal/dia. 3/EBN = 0,05861 x Consumo + 0,310794 x Ganho (Importância relativa = 73,75\%) 
As taxas de recuperação de helmintos adultos, em relação ao inóculo, variaram de 0,4 à $1,7 \%$ e de 0,2 à $0,5 \%$, para os grupos imunossuprimidos e não-imunossuprimidos, respectivamente. As espécies recuperadas incluíram $H$. contortus, na porção gástrica do trato digestivo, e T. colubriformis, no intestino delgado (tabela 2). Em outro estudo, sobre a duração da infecção de gerbis com T. colubriformis, verificou-se decréscimo no número de ovos eliminados nas fezes após 31 dias da infecção e taxas de recuperação em relação ao inóculo de
0,29 à $1,43 \%$ no dia 315 e de 0,142 à $0,428 \%$ no dia 350 após a infecção ${ }^{3}$. A observação, no presente estudo, de um maior número de helmintos adultos nos animais imunossuprimidos $(\mathrm{P}<0,05)$ está de acordo com dados da literatura ${ }^{7}$. A presença de espécimes adultos de nematódeos de ovinos no trato digestivo dos gerbis sugere, em concordância com Ziam et al. ${ }^{14}$ e Conder et al. ${ }^{2}$, que esse animal constitui modelo experimental adequado a ser tomado como referência para o estudo de verminoses que acometem pequenos ruminantes,

Tabela 2 - Espécies de nematódeos de ovinos recuperados no estômago e intestino delgado à necropsia de gerbis (Meriones unguiculatus). Campos dos Goytacazes, Junho de 2005

Nematódeos adultos

\begin{tabular}{|c|c|c|c|c|c|c|c|c|}
\hline \multirow[t]{2}{*}{ Gerbis } & \multirow[t]{2}{*}{ Tratamento } & \multicolumn{3}{|c|}{ Haemonchus contortus } & \multicolumn{3}{|c|}{ Trichostrongylus colubriformis } & \multirow[t]{2}{*}{ Total } \\
\hline & & Machos & Fêmeas & Total & Machos & Fêmeas & Total & \\
\hline 1 & $l^{b}$ & 0 & 2 & 2 & 0 & 2 & 2 & 4 \\
\hline 2 & I & 2 & 3 & 5 & 4 & 7 & 11 & 16 \\
\hline 3 & I & 3 & 3 & 6 & 5 & 6 & 11 & 17 \\
\hline 4 & I & 0 & 1 & 1 & 2 & 3 & 5 & 6 \\
\hline 5 & I & 2 & 6 & 8 & 4 & 5 & 9 & 17 \\
\hline Total & & 7 & 15 & 22 & 15 & 23 & 38 & 60 \\
\hline Percentual $^{a}$ & & 0,7 & 1,5 & 2,2 & 1,5 & 2,3 & 3,8 & 6,0 \\
\hline 6 & $\mathrm{NI}^{\mathrm{C}}$ & 2 & 1 & 3 & 0 & 0 & 0 & 3 \\
\hline 7 & $\mathrm{NI}$ & 0 & 1 & 1 & 2 & 2 & 4 & 5 \\
\hline 8 & $\mathrm{NI}$ & 0 & 0 & 0 & 0 & 2 & 2 & 2 \\
\hline 9 & $\mathrm{NI}$ & 1 & 1 & 2 & 0 & 0 & 0 & 2 \\
\hline 10 & $\mathrm{NI}$ & 0 & 0 & 0 & 0 & 0 & 0 & 0 \\
\hline Total & & 3 & 3 & 6 & 2 & 4 & 6 & 12 \\
\hline Percentual $^{a}$ & & 0,3 & 0,3 & 0,6 & 0,2 & 0,4 & 0,6 & 1,2 \\
\hline
\end{tabular}

${ }^{a}$ Helmintos recuperados em relação ao inóculo de 1,0 × $10^{3}$ larvas obtidas a partir de infecção mista

${ }^{\mathrm{b}}$ Animal imunossuprimido com 4,0mg $(0,1 \mathrm{ml})$ de metilprednisolona em doses com intervalo de 21 dias

c Animal inoculado com $0,1 \mathrm{ml}$ de solução fisiológica (placebo) 
inclusive na avaliação in vivo da atividade de antihelmínticos de amploespectro, visto que oprotocolo utilizado envolveu dois gêneros com sítios de fixação distintos. Mesmo que a imunossupressão tenha colaborado para o maior sucesso da infecção, novas pesquisas devem ser realizadas a fim de se verificar se a baixa taxa de recuperação de helmintos está associada à resistência do hospedeiro ou à interação dos parasitas envolvidos.

\section{Conclusões}

A imunossupressão em gerbis causou efeito sobre a EBN retardando o desenvo-lvimento deste. Os gerbis foram capazes de albergar $H$. contortus e T. colubriformis no estômago e intestino delgado, respectivamente, quando infectados experimentalmente.

Ainfecçãocomlarvas infectantes de espécies de nematódeos de ovinos não influenciou a EBN dos animais.

Novos estudos devem ser realizados para verificar a capacidade de gerbis em albergar nematódeos de ovinos em seu trato gastrintestinal e o efeito de infecções com uma única espécie sobre o seu desempenho.

\section{Experimental infection of jirds (Meriones unguiculatus) with larve of sheep nematodes: bio-nutritional efficiency}

\section{Abstract}

The gastrointestinal parasitism by nematodes constitutes an important cause of damages to the health and of economical losses in the production of small ruminants. Studies with nematodes of ruminants using jirds (Meriones unguiculatus) as a laboratorial model become more appropriate when the animals are immunossupressed. Corticosteroids are drugs quite used in the immunossuppression of biotery animals. The bio-nutritional efficiency index is considered an effective parameter to evaluate the effect of treatments on the animal nutritional performance. The objective of this work was to evaluate the bio-nutritional efficiency index of jirds immunossupressed and infected experimentally with larves of sheep nematodes. The results indicated that the animals that received the drug presented a major number of nematodes in necropsia in relation to the group of animals just infected. There was not harmful effect of infection on the performance of animals. The noninfected animals and treated with the drug methylprednisolone had a performance significantly lower than the noninfected animals and without drug application.
Key-words: Jirds. Nematode. Immunossupression. Bio-nutritional efficiency.

\section{Referências}

1 ECHEVARRIA, F; et al. The prevalence of anthelmintic resistance in nematode parasites of sheep in Southern Latin América : Brazil. Veterinary Parasitoogy., v. 62, n. 3-4, p.199-206, 1996.

2 CONDER, G. A. E; et al. Cocurrent infections with the ruminant nematodes Haemonchus contortus and Trichostrongylus colubriformis in jirds, Meriones unguiculatus, and use of this model for anthelmintic studies. Journal of Parasitology., v. 77, n. 4, p. 621 623, 1991.

3 ZIAM, H.; PANDEY, V. S. Duration of infection of jirds (Meriones unguiculatus) with Trichostrongylus colubriformis. Journal of Parasitology, v. 86, n. 4, p. 893-896, 2000.
4 COUTINHO, C. Infecções experimentais no coelho (Oryctolagus cuniculus L.) com nematódeos gastrintestinais de ovinos, bovinos e eqüinos 1983.70p. Dissertação (Mestrado), Universidade Federal Rural do Rio de Janeiro, Itaguaí, 1983.

5 GRATION, K.A.F. A new anthelmintic assay using rats infected with Trichostrongylus colubriformis. Veterinary Parasitology, v. 42, n. 3-4, p. 273-279, 1992.

6 ZIAM, H. et al. Drug-abbreviated infections of Trichostrongylus colubriformis and development of immunity in jirds (Meriones unguiculatus). Parasitology Research, v. 86, n. 8, p. 647-654, 2000.

7 ZIAM, H. et al. Biological parameters of Trichostrongylus colubriformis in Meriones unguiculatus. Veterinary Parasitology, v. 81, n.4, p. 309-322, 1999. 
8 GEERTS, S.; KUMAR, V.; BRANDT, J. R. A preliminary report on Meriones unguiculatus as an experimental host for Taenia saginata metacestodes. Veterinary Research Communication., v. 5, n. 3, p. 301-304, 1982.

9 KERLIN, R. L.; NOLAN, T. J.; SCHAD, G. A. Strongyloides stercoralis: histopathology of uncomplicated and hyperinfective strongyloidiasis in the Mongolian gerbil, a rodent model for human strongyloidiasis. International Journal for Parasitology, v. 25, n. 4, p. 411-420, 1995.

10 TAYLOR, M. D.; PALMER, G. C.; CALLAHAN, A. $S$. Kinetics of GTP-modulation of adenylate cyclase in gerbil cerebral cortex after bilateral ischemia. Journal of Neuroscience Research, v. 12, n. 4, p.615-621, 1984.

11 BRAUGHLER, J. M.; LAINER, M. J. The effects of large doses of methylprednisolone on neurologic recovery and survival in the Mongolian gerbil following three hours of unilateral carotid occlusion. Central Nervous System Trauma, v. 3, n. 2, p.153-162, 1986.

12 DETMANN, E.; et al. Aplicação comparativa de diferentes índices de avaliação da eficiência produtiva em experimentos com aves de corte. In: Reunião Anual da Sociedade Brasileira de Zootecnia, 41. 2004, Campo Grande. Anais... Campo Grande: SBZ, 2004 (no prelo).

13 FRAZÃO-TEIXEIRA, E. et al. Eficiência bionutricional de gerbis (Meriones unguiculatus) experimentalmente infectados com oocistos de Cystoisospora felis obtidos de fezes de gatos naturalmente infectados. Revista Universidade RuralSérie Ciências da Vida, v. 23, n. 1, p.11-12. Suplemento 2003.

14 SNEDECOR, G. W.; COCHRAN, W. G. Statistical methods. 8. ed. lowa: lowa University Press, 1989. 503 p.

15 BOOTH, N. H., McDONALD, L. E. Farmacologia e berapêutica em veterinária. 6 . ed. Rio de Janeiro: Guanabara Koogan. 1992. 997 p. 